\title{
EchoGéo
}

$18 \mid 2011$

Varia

\section{Les géographes et le patrimoine}

\section{Anne Hertzog}

\section{(2) OpenEdition}

Journals

Édition électronique

URL : https://journals.openedition.org/echogeo/12840

DOI : 10.4000/echogeo. 12840

ISSN : 1963-1197

\section{Éditeur}

Pôle de recherche pour l'organisation et la diffusion de l'information géographique (CNRS UMR 8586)

\section{Référence électronique}

Anne Hertzog, «Les géographes et le patrimoine », EchoGéo [En ligne], 18 | 2011, mis en ligne le 05 décembre 2011, consulté le 31 juillet 2021. URL : http://journals.openedition.org/echogeo/12840 ; DOI : https://doi.org/10.4000/echogeo.12840

Ce document a été généré automatiquement le 31 juillet 2021.

EchoGéo est mis à disposition selon les termes de la licence Creative Commons Attribution - Pas d'Utilisation Commerciale - Pas de Modification 4.0 International (CC BY-NC-ND) 


\title{
Les géographes et le patrimoine
}

\author{
Anne Hertzog
}

\section{Le patrimoine : l'émergence d'un objet dans les champs de recherche des géographes, révélateur d'une géographie conçue comme science de l'espace des sociétés}

1 Le patrimoine est devenu un objet commun des géographes. Dans un article de 2007, Vincent Veschambre montre clairement l'émergence des problématiques patrimoniales dans la géographie française contemporaine durant les années 1990, tout en soulignant l'entrée tardive et décalée des géographes dans « le concert patrimonial » par rapport à d'autres sciences sociales. Suite aux premières réflexions au milieu des années 1990 par Guy Di Méo sur le patrimoine urbain (ZPPAU), un certain nombre de colloques organisés par des géographes (Regards croisés sur le patrimoine dans le monde à la fin du XXe siècle, par M. Gravari-Barbas et S. Guichard Anguis en 1999) ancrent la problématique dans les approches géographiques au début des années 2000, comme en témoigne le nombre croissant de thèses, articles et publications. Cet ancrage se fonde sur la volonté d'aborder le patrimoine dans ses dimensions territoriales, sociales et politiques, comme un révélateur de la transformation des sociétés et des dynamiques spatiales. Ainsi, comme le rappelle Vincent Veschambre, le développement d'approches privilégiant la notion de "patrimonialisation» traduit la "volonté d'envisager un processus social de reconnaissance de certains héritages plutôt que les éléments patrimonialisés en tant que tels ». Ainsi, les géographes développent des approches du patrimoine insistant plus sur les « logiques sociales à l'œuvre (les différents groupes sociaux impliqués, les politiques adoptées, les constructions territoriales...) que sur l'objet patrimonial lui-même ".

Qu'ils s'intéressent aux héritages maritimes ou aux villes mexicaines, les géographes développent quelques grands questionnements, oscillant entre une interprétation du patrimoine comme "bien commun ", facteur de production de lien social et spatial et une autre étudiant le patrimoine et la patrimonialisation comme expression de 
rapports de domination voire d'une "violence symbolique» (Veschambre, 2008) produisant des inégalités et de l'exclusion. Les recherches de géographes insistent sur le lien entre patrimoine et constructions identitaires (Di Méo, Gravari-Barbas), envisagent le patrimoine comme révélateur de l'appropriation inégalitaire et conflictuelle de l'espace, ou encore comme légitimation des groupes sociaux. A travers des approches privilégiant les acteurs, certains travaux insistent sur les conflits liés à l'appropriation du patrimoine, les conflits liés à la patrimonialisation, les conflits qui s'inscrivent au sein des espaces patrimoniaux, qu'ils soient urbains ou non (Melé, 2005; Laslaz, sur les parcs naturels). La réflexion sur les enjeux de la production et de la valorisation patrimoniale des espaces, notamment dans la perspective d'une géographie du tourisme, s'affirme également avec les travaux d'Olivier Lazarrotti, tandis que se développent des réflexions sur la place du patrimoine dans les projets urbains et plus largement dans l'organisation et l'aménagement des espaces. Ainsi, dans des perspectives renouvelées, les chercheurs étudient les logiques des processus de patrimonialisation mais s'intéressent également aux freins et aux résistances, témoignant des rapports complexes des sociétés à leurs espaces et à leurs passés. La nécessité de mieux préciser les concepts liés à la patrimonialisation et à la notion même de patrimoine se fait également ressentir dans les approches contemporaines, notamment dans le dialogue avec d'autres sciences sociales (histoire, ethnologie..), par exemple autour de la distinction entre mémoire et patrimoine (voir l'entretien avec Etienne Verkindt dans ce dossier).

3 Résumant la diversité des travaux, Vincent Veschambre distingue trois grands types d'approches géographiques du patrimoine: « une approche en terme d'aménagement et de développement des territoires, sous l'angle touristique notamment, une approche en terme de paysage, de représentation et d'environnement, associée plutôt à la géographie sociale et à la géographie urbaine, une approche en terme d'identité, associée fortement à la géographie culturelle et dans une moindre mesure à la géographie historique ". Ainsi, l'intérêt croissant des géographes pour le patrimoine émane de certains renouvellements disciplinaires et d'une interaction croissante avec les autres sciences sociales (colloques, séminaires ou équipes de recherches pluridisciplinaires). Les premiers travaux intégrant la notion de patrimoine émanent de géographes se reconnaissant dans les approches sociales ou culturelles qui se développent au cours des années 1980, 1990. Ainsi, Guy Di Méo ou Maria Gravari Barbas (architecte et géographe) proposent dès le début des années 1990 des approches conceptuelles sur le lien patrimoine et territoire. Le patrimoine est donc mobilisé par une géographie questionnant le sens et la valeur des lieux, les constructions identitaires et les représentations des acteurs. Elle s'inscrit tout autant dans le renouvellement de l'approche sociale en géographie, la patrimonialisation étant alors envisagée comme facteur de différenciation sociale des espaces urbains (ségrégation, gentrification) tant dans les pays du Nord que du Sud.

4 Les approches géographiques contemporaines $\mathrm{du}$ patrimoine et de la patrimonialisation s'inscrivent cependant dans des filiations qui témoignent d'évolutions touchant également d'autres champs de la géographie: ainsi, le renouvellement d'une géographie politique abordant les registres symboliques de l'action publique (Lussault) et renouvelant l'étude des politiques territoriales et des jeux d'acteurs. Par ailleurs, l'affirmation d'une géographie plus sensible au temps, envisageant la longue durée des constructions territoriales, la résilience des systèmes spatiaux dans la perspective d'une géohistoire renouvelée, conduit certains géographes 
à repenser le patrimoine comme une construction sociale d'un rapport au temps (Poncet, Djament-Tran). Enfin, les travaux questionnant la patrimonialisation de la nature (patrimoine «naturel») s'inscrivent dans le renouvellement des approches de la nature au cours des années 1980 au sein d'une géographie physique en pleine mutation: dans une perspective environnementaliste envisageant non pas la nature pour elle-même mais recentrée sur les "formes d'appropriation sociale de la nature " (Bertrand), certains géographes (rôle des biogéographes) réorientent leurs problématiques vers l'étude d'une "nature conforme aux logiques de la société " (Veyret). C'est bien d'une socialisation de la nature à travers sa patrimonialisation dont il est question dans l'entretien avec Jean-Paul Amat publié dans ce dossier. La question du patrimoine «naturel » est cependant souvent envisagée comme une problématique en soi dans les approches géographiques du patrimoine, cette notion étant souvent associée aux objets (bâtis, collections) ou aux héritages culturels matériels ou immatériels fabriqués par les sociétés (villes). L'article de Vincent Veschambre de 2007 consacré aux approches géographiques du patrimoine n'évoque que très indirectement la nature témoignant implicitement d'un clivage disciplinaire et une distinction entre nature et culture. L'entretien avec Jean-Paul Amat peut de ce point de vue également conduire à un certain nombre de questionnements sur la définition même des catégories patrimoniales et la façon de les « lire ».

5 L'émergence de la problématique patrimoniale dans les recherches géographiques s'inscrit donc dans le profond renouvellement de la géographie depuis quelques décennies. Les géographes contemporains s'en saisissent aujourd'hui comme d'un objet permettant l'effacement des frontières internes de la géographie, et de ce point de vue, les travaux sur le patrimoine constituent en retour un facteur de renouvellement de la géographie. Par exemple, Vincent Veschambre fait de la problématique patrimoniale le point d'ancrage d'une approche renouvelée des inégalités sociales et des rapports de domination, articulant étroitement points de vue de la géographie culturelle et de la géographie sociale. Les réflexions menées par Jean-Paul Amat sur le patrimoine forestier témoignent également d'une tentative de dépassement de certaines frontières disciplinaires.

6 Soulignons l'articulation de ces recherches à la multiplication des formations universitaires dans les domaines de la culture et du patrimoine ces dernières décennies, dans le cadre de Masters de géographie spécialisés en tourisme ou en aménagement/développement territorial (dans le même temps, les problématiques géographiques semblent entrer plus difficilement dans les formations spécialisées dans le domaine du patrimoine). La multiplication des cursus de géographie intégrant les problématiques patrimoniales, outre qu'ils alimentent la recherche (thèses), témoigne de manière évidente, par leur orientation professionnelle (voir l'entretien avec Etienne Verkindt sur ce point), de la "soif» de patrimoine dans nos sociétés et de l'inscription des problématiques géographiques dans un contexte social, politique, économique et culturel particulier.

\section{La réflexion géographique en réponse à une demande sociale et politique grandissante}

7 Les mutations sociétales - modernisation, urbanisation, montée du tourisme, généralisation des mobilités, et les recompositions territoriales liées à la mondialisation 
et aux redistributions des pouvoirs (décentralisations, nouveaux territoires) ont contribué au renouvellement de la notion de patrimoine et à son extension typologique, chronologique et spatiale depuis longtemps identifiées par les sciences sociales et particulièrement par les géographes (Gravari-Barbas, Veschambre). L'enjeu économique et la construction de l'attractivité des territoires, dans un contexte concurrentiel, conduisent un certain nombre d'acteurs à valoriser des ressources territoriales - patrimoniales - impliquant la construction de modalités sans cesse renouvelées de médiation des patrimoines.

8 L'intégration des problématiques patrimoniales dans les approches géographiques est à l'évidence le produit de ce contexte social marqué par le "goût du passé ", où les questions patrimoniales et mémorielles deviennent des préoccupations sociales et politiques, voire se muent en injonction à se souvenir ou à conserver. Aussi, les conceptions d'organisation et d'aménagement des espaces où s'imposent les logiques de conservation, de protection, de patrimonialisation tendent-elles à s'intensifier et à se mondialiser - notamment sous l'effet d'un discours normatif porté par les organisations internationales comme l'Unesco. L'entretien mené avec Jean-Paul Amat s'en fait largement l'écho.

Aussi, l'implication grandissante des géographes dans la réflexion sur le patrimoine estelle une réponse à ce contexte et à une demande sociale et politique croissante en matière de définition et de valorisation des patrimoines. Les dynamiques sociospatiales du local au mondial entrainent en effet leur redéfinition permanente et des questionnements sans cesse renouvelés sur leur place dans les sociétés contemporaines : en témoignent les interrogations sur les modalités de définitions et de mises en valeur des héritages à transmettre dans le contexte de la ville postcoloniale ou de la ville post-industrielle.

De même, la mutation des cadres sociaux, politiques et territoriaux de définition des patrimoines - l'État, la Nation - sous l'effet de la mondialisation et de la métropolisation, des nouveaux modes de gouvernance, questionne la pertinence des échelles de la patrimonialisation tant du côté des chercheurs en sciences sociales, que du côté des acteurs politiques ou des professionnels du patrimoine, comme le montre la recrudescence des colloques sur ces questions (les Entretiens du Patrimoine 2011 questionnaient ainsi les redéfinitions possibles des patrimoines urbains dans le contexte de métropolisation). L'entretien avec Etienne Verkindt renvoie à la question de l'européanisation des constructions patrimoniales à travers l'émergence de politiques patrimoniales transnationales dans le cadre de la politique régionale transfrontalières de l'Union Européenne. À un autre niveau d'échelle, l'émergence de la notion de patrimoine mondial de l'Unesco renvoie également à la question des échelles et des dynamiques spatiales des processus de patrimonialisation, de même qu'elle questionne également la notion de patrimoine comme "bien commun", suscitant depuis quelques décennies, de nombreuses réflexions tant dans le champ du politique que dans les approches scientifiques. L'entretien avec Jean-Paul Amat témoigne de l'implication des géographes dans les processus de classement Unesco ou au sein d'organisation internationales comme l'Icomos. 


\section{Les géographes coproducteurs de patrimoines, experts de la valorisation des patrimoines}

11 On assiste depuis quelques décennies à une institutionnalisation et à une professionnalisation de la connaissance et de la valorisation de ce patrimoine, faisant émerger de nouvelles figures professionnelles intervenant dans les dispositifs de sélection et de médiation des héritages matériels et immatériels (inventaires, circuits culturels, musées, centres d'interprétation...), liées à la formalisation progressive des politiques patrimoniales et à leur territorialisation à différents niveaux d'échelle. Ainsi, de nombreux géographes sont engagés aux côtés des acteurs publics au sein des projets d'aménagement parce que leur approche répond aux préoccupations de valorisation ou de structuration des espaces patrimoniaux dans la perspective de développement territorial, ce dont témoignent les deux entretiens de ce dossier. Ils interviennent notamment au sein des comités scientifiques ou de commissions de réflexion désormais inhérentes aux politiques patrimoniales territorialisées, du moins dans le cas français (condition d'obtention de financements ou des labellisations, fonctionnement des dispositifs). Aussi les géographes sont-ils présents dans les comités scientifiques ou les équipes pluridisciplinaires des PNR (Ballons des Vosges, Gâtinais, voir entretien avec Jean-Paul Amat). Ils sont également amenés à intervenir dans les comités scientifiques des musées : certains ont activement participé à la constitution d'écomusées aux côtés des ethnologues ou des historiens (écomusée du Beauvaisis) ou des musées de territoires (musées de ville comme celui de la Communauté d'agglomération de St Quentin en Yvelines par exemple). L'expérience de Jean-Paul Amat montre qu'ils peuvent être impliqués de manière peut-être plus inattendue, dans les musées d'histoire. Certes, les géographes sont absents de nombreuses institutions et projets patrimoniaux (on peut penser aux divers musées de l'Europe qui se sont faits sans l'apport réflexif de géographes) - renvoyant à un déficit de légitimité de la géographie en raison de son insertion plus tardive à la fois au sein des sciences sociales et dans le champ des études patrimoniales longtemps dominées par les historiens, historiens de l'art ou les ethnologues (Veschambre). Leur implication croissante peut alors s'interpréter comme le signe d'une légitimité scientifique grandissante, mais peut-être surtout comme celui de leur capacité à répondre aux préoccupations sociales et politiques en termes d'aménagement et de valorisation de patrimoines territorialisés.

La participation au sein d'un comité signifie reconnaissance des compétences. Cependant, la "spécialité " n'est pas le seul critère de la composition des comités, révélant la force des réseaux professionnels et amicaux. Les expériences de Jean Paul Amat au sein des comités scientifiques de plusieurs projets muséographiques le montrent. Ces comités sont des espaces d'échanges et d'interaction entre acteurs, souvent issus de sphères différentes sur le plan disciplinaire ou professionnel. En effet, leur composition est aussi révélatrice du degré d'ancrage local des projets et des jeux d'acteurs locaux dans les processus de patrimonialisation. La tendance est à l'ouverture disciplinaire et à l'interaction entre acteurs locaux et intervenants extérieurs, voire internationaux (patrimoine Unesco). Aussi, ces comités sont-ils des espaces de cohabitation par excellence entre représentants des sociétés locales - bénévoles ou historiens amateurs - et de divers professionnels de la culture ou du patrimoine, mais aussi entre politiques et scientifiques, ce que ne manquent pas de souligner J.-P. Amat et $\mathrm{E}$. Verkindt, insistant tous deux sur la capacité du géographe à être un « facilitateur » 
dans le dialogue entre sciences. Ainsi, la vision d'une géographie, science carrefour, souvent perçue comme relevant d'un déficit de légitimité scientifique dans le champ de la recherche semble ici un atout valorisé et perçu de manière positive.

Les entretiens menés avec le Pr. Jean-Paul Amat, professeur de biogéographie, et Etienne Verkindt, doctorant en géographie, donnent un aperçu de la diversité des modalités d'expertises apportées par les géographes dans les processus de patrimonialisation. Ils montrent comment la réflexion du géographe sur le patrimoine se construit dans l'échange disciplinaire, en interaction avec la demande sociale et le politique.

Le géographe est engagé dans le processus de désignation du patrimoine, autrement dit il est acteur de la « fabrique » du patrimoine (Heinich). En effet, il invente de nouvelles catégories spatiales territoriales comme en témoigne Etienne Verkind. Celui-ci importe une catégorie présente dans le champ du politique (politique mémorielle de la Flandre Occidentale, filiation avec la notion de " paysages culturels évolutifs » de l'Unesco) dans le champ du scientifique (thèse). Laquelle catégorie est ensuite réinvestie dans le processus de labellisation Unesco qui lui attribue de nouveaux contours en fonction des critères de définition dans le cadre de la labellisation. L'entretien avec Jean Paul Amat témoigne également du rôle du géographe dans la fabrique du patrimoine à travers la participation au plan de gestion de la forêt de Verdun et la définition d'un zonage classant les espaces forestiers en fonction de leur «valeur " patrimoniale. L'action de désignation des espaces patrimoniaux par l'élaboration de zonages est fondée sur une méthodologie complexe (telle qu'elle est par exemple décrite par Etienne Verkindt) où interviennent les démarches et méthodes des géographes (analyse paysagère). La mobilisation d'outils comme les SIG ou la cartographie est une autre modalité de l'apport des géographes à l'expertise patrimoniale comme le montre l'expérience de Jean-Paul Amat dans la réalisation de "cartes patrimoniales» ou le travail de l'université de Gand évoqué par Etienne Verkindt. Ces deux exemples renvoient à la mobilisation des outils géographiques (SIG) ou cartographiques dans l'identification et l'inventaire des patrimoines, témoignant également de la territorialisation des politiques patrimoniales.

15 Le géographe intervient également dans l'étude et la connaissance des espaces dont la valeur patrimoniale est reconnue par d'autres (acteurs politiques): la connaissance qu'il apporte sur le territoire vient alors appuyer ou justifier la « valeur " patrimoniale du lieu. Les exemples de Langres ou de la forêt de Verdun développés par Jean-Paul Amat le montrent; on peut également penser aux études demandés à certains géographes dans le cadre de la constitution de musées de territoires.

16 Comme le montre l'engagement de Jean-Paul Amat, la réflexion du géographe peut aussi se développer à d'autres niveaux, comme la réflexion sur l'organisation et la structuration des lieux patrimoniaux, notamment dans une perspective de valorisation touristique. Rendre un lieu patrimonial « lisible » par la structuration de jalonnements ou d'itinéraires, mieux penser l'intégration d'un musée dans son territoire, telles sont les questions mobilisant l'intervention des géographes dans un contexte de territorialisation du patrimoine.

17 Enfin, dans le cas de Jean Paul Amat, l'expertise menée au sein de la réserve de biosphère et du PNR du Gâtinais relève essentiellement du diagnostic territorial et de la réflexion sur la conciliation des enjeux d'aménagement et de conservation d'une nature patrimonialisée. 


\section{BIBLIOGRAPHIE}

Autrepart, 2005. Inventer le patrimoine moderne dans les villes du Sud. Autrepart, $\mathrm{n}^{\circ} 33$.

Di Méo G., 1995. Patrimoine et territoire, une parenté conceptuelle. Espaces et Sociétés, $\mathrm{n}^{\circ} 78$, p. 16-33.

Djament-Tran G., 2011. Rome éternelle, Belin.

Gravari-Barbas M., 1996. Le "sang" et le "sol" : le patrimoine, facteur d'appartenance à un territoire urbain. Géographie et culture, n²0, p. 55-68.

Gravari-Barbas M., Guichard-Anguis S. (dir.), 2003. Regards croisés sur le patrimoine dans le monde. Paris, Presses de l'Université de Paris-Sorbonne, 958 p.

Gravari Barbas M., Veschambre V., 2003. Patrimoine : derrière l'idée de consensus les enjeux d'appropriation de l'espace et des conflits. In P. Melé, C. Larrue, M. Rosemberg (coord.), Conflits et territoires, Tours, PUFR, MSH “Villes et territoires”, p. 67-82

Gravari-Barbas M. (dir.), 2005. Habiter le patrimoine : enjeux, approches, vécu. Rennes, PUR, 618 p.

Guichard-Anguis S., Héritier S., 2009. Le patrimoine naturel : entre ressource et culture. Géographie et Culture, 66.

Heinich N., 2009. La fabrique du patrimoine. De la cathédrale à la petite cuillère, Editions de la Maison des Sciences de l'Homme, collection Ethnologie de la France.

Héritier S., Laslaz L. (coord.), 2008. Les parcs nationaux dans le monde. Paris : Ellipses.

Lazzarotti O., Violier Ph. (coll.), 2007. Tourisme et patrimoine. Un moment du Monde. Presses Universitaires d'Angers, $248 \mathrm{p}$.

Lazzarotti O., 2011. Patrimoine et tourisme, Belin Sup, 302 p.

Lussault M. 1997. Des récits et des lieux : le registre identitaire dans l'action urbaine. Annales de Géographie, n 597, p. 522-530

Melé P., 2005. Conflits patrimoniaux et régulations urbaines. Travaux et Documents ESO, $\mathrm{n}^{\circ} 23$, p. 51-57

Norois. Patrimoine et environnement, les territoires du conflit. Norois, 185, t 47, 174 p.

Poncet P., 2004. Du patrimoine national à la "société de conservation. Pouvoirs locaux, $\mathrm{n}^{\circ} 63$, décembre 2004.

Tomas F., 2004. Les temporalités du patrimoine et de l'aménagement urbain. Géocarrefour, vol. 79, 3/2004, p. 197-210.

Veschambre V., 2007. Patrimoine : un objet révélateur des évolutions de la géographie et de sa place dans les sciences sociales. Annales de géographie, 2007/4 n 656, p. 361-381.

Veschambre V., 2008. Traces et mémoires urbaines, enjeux sociaux de la patrimonialisation et de la démolition. Presses universitaires de Rennes. 


\section{AUTEUR}

\section{ANNE HERTZOG}

Anne Hertzog, hertzog.anne@wanadoo.fr, est Maître de conférences à l'Université de Cergy Pontoise. Sur les questions patrimoniales elle a notamment publié :

- Hertzog A., 2006. Les musées de la Grande guerre en Picardie et leurs territoires. La patrimonialisation de la guerre dans une région en recomposition : approche géographique d'un phénomène contemporain. In La géographie militaire de la Picardie, dir. par Ph. Nivet et Ph. Boulanger, Encrage édition.

- Hertzog A., 2007. Territoire patrimonialisé, culture territorialisée : musées et ressource territoriale. In Gumuchian H., Pecqueur B., La ressource territoriale, Economica-Anthropos, 2007. - Hertzog A., Poulard F, 2008. Les échanges internationaux comme point d'ancrage de la modernisation et de la diversification des musées français. In Patrimoine et Mondialisation, Groupe de Recherche sur les Musées et les patrimoines, L'Harmattan.

- Hertzog A., 2009. Les modèles de musées : spatialités et temporalités, étude des dynamiques de localisation en Picardie. In De nouveaux modèles de musées? Formes et enjeux des créations et rénovations de musées en Europe. XIXe-XXIe siècles, L'Harmattan, coll. Patrimoines et Sociétés, p. $163-178$.

- Hertzog A., 2010. La valorisation du patrimoine de la Première guerre mondiale en Picardie. La fabrique d'une ressource culturelle territoriale. In Fournier L-S., Bernié-Boissard C., Crozat D., Chastagner Cl., Développement culturel et territoires, L'Harmattan, p 189-208. 